Relations industrielles

Industrial Relations

\title{
Marbach, Valérie, Évaluer et rénumérer les compétences
}

\section{Laurent Bélanger}

Volume 55, numéro 4, 2000

URI : https://id.erudit.org/iderudit/051361ar

DOI : https://doi.org/10.7202/051361ar

Aller au sommaire du numéro

Éditeur(s)

Département des relations industrielles de l'Université Laval

ISSN

0034-379X (imprimé)

1703-8138 (numérique)

Découvrir la revue

Citer ce compte rendu

Bélanger, L. (2000). Compte rendu de [Marbach, Valérie, Évaluer et rénumérer les compétences]. Relations industrielles / Industrial Relations, 55(4), 778-778.

https://doi.org/10.7202/051361ar

Tous droits réservés (C Département des relations industrielles de l'Université Laval, 2000
Ce document est protégé par la loi sur le droit d'auteur. L’utilisation des services d'Érudit (y compris la reproduction) est assujettie à sa politique d'utilisation que vous pouvez consulter en ligne.

https://apropos.erudit.org/fr/usagers/politique-dutilisation/ 


\section{Évaluer et rémunérer les compétences}

par Valérie MARBACH, Paris : Éditions d'organisation, collection développement et emploi, 1999, 193 p., ISBN 2-7081-2288-6.

Ce petit ouvrage est un effort pour faire le point sur les difficultés inhérentes à la mesure des compétences et à l'intégration de la reconnaissance des compétences aux systèmes de rémunération actuels. L'ouvrage comprend donc deux parties qui explorent chaque volet.

Une première partie, comprenant six chapitres, aborde la notion de compétences, plus particulièrement les dimensions de «savoir », de «savoir-faire » et de « savoir-être » inhérentes à cette notion, l'établissement d'une hiérarchie de compétences et les difficultés reliées à leur évaluation. En reprenant le débat sur la classification et la qualification, l'auteure s'interroge sur une substitution éventuelle du concept de compétence à celui de qualification. Par la suite, elle décrit la place et l'importance d'une référence au critère de compétence dans un processus d'évaluation et de hiérarchisation des emplois. À ce sujet, elle invite à une réflexion qui prend en compte l'ensemble de la situation professionnelle du salarié en suivant la gradation suivante : de l'emploi traditionnel ou poste de travail taylorien à l'emploi à géométrie variable pour passer de ce dernier à l'emploi recomposé qui renvoie à la situation professionnelle personnalisée. Cette partie se termine par une description de la situation japonaise où l'emploi à vie, surtout dans les grandes entreprises, permet d'illustrer la distinction que l'auteure tente d'établir entre une gestion qualifiante, fortement axée sur la rotation des postes et une organisation qualifiante qui elle désigne plutôt une certaine forme d'enrichissement du travail.

La deuxième partie traite de la prise en compte des compétences dans les systèmes de rémunération. Pour ce faire, l'auteure reprend d'abord les caractéristiques d'un système de rémunération en mettant en évidence les dimensions autres qu'économique, à savoir la dimension sociale et la dimension managériale pour aborder par la suite le phénomène d'individualisation des salaires qui s'installe lorsqu' on recherche la reconnaissance des compétences dans les pratiques de rémunération. Elle propose donc une typologie des systèmes de rémunération, autres que celles qui sont issues des grands cabinets conseil nordaméricains et qui comprend à son tour cinq modèles allant de l'emploi de type taylorien à celui où l'on prendrait en compte les compétences potentielles de la personne. Trois de ces modèles renvoient à la notion de compétences utilisées et deux à celle de compétences utilisables. Des exemples concrets sont fournis pour illustrer la nature et le fonctionnement de chacun de ces modèles et le dernier, celui de la prise en compte des compétences potentielles, serait celui qui traduit le mieux la notion de gestion par les compétences.

Le débat sur la classification, la qualification et les compétences n'a pas un caractère très percutant en contexte nord-américain où le concept de qualification est encore intimement associé à la description formelle des postes et sert d'ingrédient important dans la détermination de leur valeur relative et leur inclusion dans une classification. Cet ouvrage est une invitation à délaisser une approche de la rémunération axée sur la qualification dans le cadre plutôt étroit des descriptions formelles de postes pour s'intéresser à des modes de rémunération qui, en plus de retenir la performance et le mérite comme critères, intégreraient des attributs de la personne en situation de travail par le biais des compétences prouvées ou utilisables. En ce faisant, il serait possible d'adopter des pratiques de rémunération qui s'inscriraient dans une logique de la compétence.

LAURENT BÉLANGER Université Laval 